\title{
The Behaviour of Cardiac Macrophages in the Steady State, Injured, and Ageing Heart: A Systematic Review
}

\author{
Susan Cai, BSc Student [1]*
}

[1] Department of Microbiology and Immunology, McGill University, Montreal, QC, Canada, H3A 0G4

*Corresponding Author: susan.cai@mail.mcgill.ca

\begin{abstract}
Introduction: Research conducted over the past decade has revealed that the heart is home to both recruited and tissueresident macrophages, both of which play a vital role in cardiac development, composition, and function. Moreover, clinical studies have demonstrated that tissue-resident macrophages contribute considerably to a variety of regulatory and housekeeping tasks in the homeostatic heart. It has been long established that following cardiac injury, macrophages work to clear the heart of debris, stimulate the regeneration of damaged tissue and stabilize the cardiac wall. However, much remains elusive about the exact nature of cardiac macrophages. Hence, this review will analyze and summarize the current literature documenting the critical roles, origins, phenotypes and biomechanisms of macrophages in cardiac homeostasis and cardiac disease.

Methods: An overarching map depicting the relationship between cardiac macrophages and the cardiac environment was assembled via a systematic review of the extant literature on the origins, phenotypes, biochemical profiles and biomechanisms of cardiac macrophages. In total, 28 works were analyzed to determine the importance of macrophages in the homeostatic, injured, and ageing heart.

Results: Research conducted over the past decade shows that the heart is home to a heterogeneous population of cardiac macrophages. Contrary to the historic perspective that all cardiac macrophages are derived from circulating blood monocytes, evidence has demonstrated that most of these macrophages are of embryonic origin. Extant literature has identified various subsets, each of which appears to be responsible for either reparative or inflammatory tasks.

Discussion: It is foreseen that developing a more comprehensive understanding of cardiac macrophages may open new doors to novel therapeutic methods for cardiac diseases and disorders. The advancement of treatment procedures post heart failure may be a vital step in lowering the frequency of periodic episodes amongst patients with chronic heart dysfunctions.

Conclusion: The observed behaviour of murine and human cardiac macrophages in various cardiac conditions has led to the development of three main perspectives: one, a macrophage's ontogeny dictates its function; two, the local cardiac tissue dictate macrophage function; and three, the nature versus nurture argument is a false dichotomy.
\end{abstract}

Keywords: cardiac immune cells; cardiac resident macrophages; macrophage phenotypes; cardiac disease; cardiac injury; cardiac homeostasis; cardiac ageing

\section{Introduction}

Historically, it has been assumed that cardiac macrophages originate solely from circulating blood monocytes. However, the recent interest in cardioimmunolgy has uncovered evidence that demonstrates otherwise [1]. This growing body of literature has revolutionized the common understanding of the nature of cardiac macrophages. As such, these emerging studies have highlighted literature gaps regarding their known characteristics, purposes, and mechanisms of action [1]. Murine and human models have demonstrated that cardiac macrophages represent a heterogeneous population of cells derived from various lineages [1]. Furthermore, evidence has determined that many cardiac macrophages develop alongside other tissue-resident macrophages found in the brain, skin, liver, kidney, and lungs during embryonic development. Embryonic-derived macrophages are longlived and replenished locally, independent of peripheral monocyte input through cell proliferation [1]. This emerging perspective deviates from the long-accepted theory wherein all cardiac macrophages originate from definitive hematopoietic progenitors located within the bone marrow and spleen; and are said to be replenished under steady-state and inflammatory conditions through monocyte recruitment [1,2]. These advancements in knowledge relied on the development and establishment of sophisticated techniques such as genetic lineage tracing and monocyte tracking [1].

Although the exact details of their varied origins continue to be elucidated, it has become apparent that 
UNDERGRADUATE RESEARCH IN NATURAL AND CLINICAL SCIENCE AND TECHNOLOGY (URNCST) JOURNAL Read more URNCST Journal articles and submit your own today at: $\underline{\text { https://www.urncst.com }}$

macrophage ontogeny critically influences their cellular behaviour. Understanding and defining their genesis is particularly important, as macrophages of distinct origins often co-exist within tissues. Fate-mapping analysis has revealed that a majority of the cardiac macrophage population is of embryonic origin, thereby making the heart amongst the small handful of organs that retain a substantial number of yolk-sac macrophages into adulthood [3-5].

Altogether, it has been collectively established that murine and human hearts contain a heterogeneous population of functionally distinct macrophages with a remarkable impact on the maintenance and repair of the cardiac environment [1]. With the development of a range of novel techniques, the possibility of conducting further research on human cardioimmunolgy has expanded. Therefore, this study aims to conduct a systematic review of the origins, functions, and characteristics of cardiac macrophages. Through the analysis and summarization of extant research, this study will assemble an overarching map illustrating what is known about cardiac macrophages and what remains unexplored.

\section{Methods}

Following PRISMA guidelines for systematic literature reviews, comprehensive literature searches were conducted in the following databases for both published and unpublished studies in the English language: PubMed, OVID, Cochrane Library, MEDLINE and MedNar. The following terms were used in a variety of combinations to generate the searches: cardiac macrophages, tissue-resident macrophages, cardiac immune cells, heart disease, homeostasis, cardiac remodeling, and cardiac repair. A total of 1,180 records were identified through database searching, however, following the removal of all duplicates, only a total of 512 records remained. From there an abstract review was used to determine the relevancy of the gathered records; those that revealed limited information on the origins, functions or characteristics of cardiac macrophages were removed from the search. Additionally, texts belonging to sub-sections of larger studies were removed from the data pool; this was done to prevent the inclusion of any partially accurate or incomplete perspectives and speculations. Furthermore, studies that examined the effects of synthetic factors such as drugs on cardiac macrophages were excluded. This is to ensure that the literature cited in the final review is specifically pertinent to the intuitive behaviour of macrophages in the steady state, ageing and injured heart. Following the abstract review, a full-text review was conducted across the remaining 104 records. Studies conducted prior to 2011 were removed from the search such that the final review may be succinct and relevant. Additionally, studies that focused on the feline or canine heart were excluded such that this review may focus on murine and human models. The final, remaining 28 records are included and summarized in the results of this review. Collectively, these findings were interpreted to form an overarching map detailing what is currently known about the identity of cardiac macrophages; recommendations for future avenues of research; what remains unknown or ill-defined about cardiac macrophages; and the limitations and uncertainties around the accepted understanding and ongoing research.

\section{Results}

A healthy human adult heart is known to consist of endothelial cells, cardiomyocytes, fibroblasts, pericytes, mesenchymal cells and various types of immune cells [6]. Evidence has shown that the heart contains a variety of immune cells, including all major leukocyte classes, namely mononuclear phagocytes, neutrophils, B cells and T cells. Moreover, they exist at frequencies that surpass those in skeletal muscle by 12-fold [7]. Interspersed throughout the cardiac environment and lodged in specific niches between cardiomyocytes is a network of resident macrophages. These macrophages make up approximately $12 \%$ of the total immune cell population found in the cardiac environment [8]. Macrophages are a part of both the innate and adaptive immune system and play a major role in vital tasks such as immune system defence, inflammation, and tissue restoration [6].

\section{Macrophages in the Steady-State Heart}

In the steady-state, tissue-resident macrophages execute a range of homeostatic functions. Observations have indicated that this includes, but is not limited to, defending against infection and the removal of damaged or dying cells [9]. Like any other macrophage (tissue-resident or otherwise), cardiac macrophages neutralize bacterial and apoptotic cells via phagocytosis. However, as the heart is rarely under the threat of a bacterial infection, these cardiac macrophages are primarily ingesting aged or dying cardiomyocytes [10]. At the turn of the last decade, many of the preliminary studies on cardiac macrophages sought to categorize these seemingly irregular immune cells on a molecular level. Through gene expression analysis, these works found that a large portion of the cardiac macrophage population closely resembled alternatively activated antiinflammatory M2 macrophages [11]. Using a combination of single marker labelling techniques and immunoblotting/immunofluorescence approaches, a number of works have identified 14 different markers on monocytes and macrophages. In particular, the discovery of the expression of specific surface markers such as Mrc1, CD163 and Lyve-1 on certain cardiac macrophages brought forth two main implications: one, that the heart could contain multiple subsets of various macrophage phenotypes; and two, certain subsets could potentially play a vital role in cardiac homeostasis $[9,12]$. Recent studies have now directly observed macrophage involvement in the stimulation of the restoration and regeneration of cardiac tissue and have since begun to establish a clearer narrative as to which mechanisms are used to trigger these reparative 
UNDERGRADUATE RESEARCH IN NATURAL AND CLINICAL SCIENCE AND TECHNOLOGY (URNCST) JOURNAL Read more URNCST Journal articles and submit your own today at: https://www.urnest.com

functions $[6,13]$. However, a definite understanding of their mechanobiology has yet to be outlined due to the variety and complexity these cells seem to posses [14]. Of the 28 records analyzed for this review, a majority described the identification of three main subsets through the varied expressions of MHC-II, CCR2 and Ly6C [1,2,5]. Although the exact protocol varied from study to study, observation and identification of the three subsets was achieved through use of CX3Cr1-GFP mice; immunofluorescence was first employed to track (and label) the expression of distinct surface proteins on macrophages; each identified subset was then purified using flow cytometry and subjected to microarray gene expression profiling [5]. The three subsets are presently classified as either a classically activated, M1 macrophage $(\mathrm{CCR} 2+)$ or an alternatively activated M2 macrophages (CCR2-) [9]. However, a handful of records have recently established the potential presence of a fourth macrophage subset that demonstrates an M4 phenotype [9]. These newly identified cells have attracted much attention due to the response mechanisms they can develop [9]. Of these three main phenotypes, evidence has demonstrated that it is the M2 phenotype that is heavily responsible for the execution of reparative behaviours in the homeostatic heart [9]. M2 macrophages are further subdivided into M2a, M2b, and M2c. M2a are stimulated with IL-4 or IL-13; while $\mathrm{M} 2 \mathrm{~b}$ are stimulated via immune complexes in combination with IL-1 $\beta$; and M2c are stimulated with IL10, TGF- $\beta$ or glucocorticoids [9]. M2a and M2c macrophages are primarily accountable for coordinating adaptive immune response, whereas M2b macrophages work to suppress inflammation [9]. However, all M2 macrophages can be identified through the lack of CCR2 expression, or in other words, are characterized as CCR2$[2,4,9,15]$.

As aforementioned, paradigm-shifting studies conducted over the past decade have demonstrated that the cardiac macrophage population is of heterogeneous ontology. Furthermore, contemporary studies have shown that most of the cardiac macrophage population is of embryonic origin $[2,7,9]$. Resident cardiac macrophages were observed to start developing as early as day 7 of embryonic development, where primitive hematopoiesis occurs, leading to the expansion of macrophages into the extraembryonic yolk sac [2,3]. Subsequently, erythromyeloid precursors emerge from the yolk sac and generate fetal macrophages $[3,16]$. A handful of these immune cells will then infiltrate the heart where they will remain in the myocardium and participate in housekeeping tasks for life [2]. This first wave of colonization is followed by a second wave of fetal liver monocytes, which will later differentiate into cardiac tissue macrophages. Finally, a third wave of post-natal bone marrow-derived monocytes will infiltrate the heart giving rise to the well-known monocytederived, M1 macrophages [2,3]. Interestingly, early studies of the neonatal heart showed that it has a remarkable capacity for tissue repair [13]. However, this protective function is observed to disappear two weeks into life-around the same time bone-marrow-derived monocytes infiltrate the heart $[3,13]$. Thus, some have interpreted these findings as an indication that macrophages of embryonic origin are inherently reparative while monocyte-derived macrophages are inherently inflammatory $[3,13]$.

Additionally, using a diphtheria toxin receptor (DTR)based, in-vivo murine system, studies have observed that post-surgical apical resection, cryoablation or myocardial infarction, the neonatal mice who efficiently regenerated the myocardium had increased levels of cardiac macrophages. Subsequent investigations using flow cytometry and genetic lineage tracing revealed that there is indeed a distinct lineage of embryo derived, resident M2 macrophages present in both the neonatal and adult hearts [13]. However, in response to injury, the neonatal heart was seen to only selectively expand the number of resident, (MHC-II, low CCR2-) M2 macrophages and did not recruit any additional CCR2+ monocytes. It is suspected that it is ultimately these resident M2 macrophages that secrete factors that aid in angiogenesis, the formation of granulation tissue and collagen deposition. Furthermore, based on how various other populations of resident macrophage operate, it is hypothesized that these MHC-II, low CCR2- macrophages will activate stem/progenitor cells in preparation for the reconstruction of damaged areas. However, this capability for selective recruitment appears to diminish as an organism ages.

The same studies that have identified a distinct difference between M1 and M2 phenotypes have also discovered that under in vitro conditions, cardiac macrophages are capable of mutually converting between phenotypes $[9,12]$. For example, M1 macrophages could switch to the M2 phenotype post-stimulation with pro-M2 factors and vice versa [9]. Hence, a handful of works have interpreted these findings as a possibility that the various macrophage phenotypes, and by extension their roles, are not inherently determined by their origins but instead rely on external factors and signals supplied by the surrounding tissue $[2,13]$. For instance, it was observed that monocytederived macrophages will exhibit CCR2+ expression upon their initial infiltration into the cardiac environment but will progressively lose said character over time. Essentially, as they age, these monocyte-derived macrophages slowly become more like embryonic, M2 macrophages on a molecular level [3].

Furthermore, in a study conducted by Nahrendorf et. al., it was demonstrated that in addition to their expected list of "housekeeping" tasks, macrophages also seem to play a role in the facilitation of electrical conduction in the heart $[8,9]$. Using macrophage reporter lines in combination with optical clearing techniques and confocal microscopy, it was demonstrated that macrophages are abundant in the atrioventricular (AV) node [9]. Moreover, it has been observed that these AV nodal macrophages intervene with cardiomyocytes through connexin-43 containing gap 
UNDERGRADUATE RESEARCH IN NATURAL AND CLINICAL SCIENCE AND TECHNOLOGY (URNCST) JOURNAL Read more URNCST Journal articles and submit your own today at: https://www.urncst.com

junctions to accelerate myocyte repolarization and electrical conduction $[9,17,18]$.

Macrophages in the Injured Heart

Typically, in response to the necrosis or degeneration of cardiomyocytes and other cardiac cell types, the myocardium will undergo inflammation, a process commonly known as myocarditis [6]. The most common causes of myocarditis are infections of either parasitic or viral origin. However, regardless of the nature of the primary trigger, the subsequent immediate immune response is always mediated by the cause itself, while the following injury to cardiac tissue is observed to be indirectly mediated via the immune system [6]. The innate immune response is essential for the elimination of viral, pathogenic, and bacterial infections and the restoration of healthy tissue $[6,12]$. Upon pathogenic entry, a cascade of inflammatory signaling sequences is triggered, thereby resulting in the production of various pro-inflammatory cytokines and chemokines. Upregulation of these cytokines and chemokines subsequently trigger macrophage polarization, thereby activating the heart's M1 macrophages. These macrophages then recruit a slew of blood monocytes, causing them to flood the heart and, overall, aggravate the inflammatory process [6,9]. Although cardiac macrophages are seen to participate heavily in evidently clear cases of inflammatory cardiac injury such as myocarditis, recent studies have also demonstrated links between these M1 macrophages and various other cardiac injuries such as myocardial ischemia and myocardial infarction.

Similar to how the M2 phenotype can be further classified, M1 macrophages can be divided into M1a or M1b. M1a macrophages are stimulated with toll-like receptors and M1b macrophages are stimulated via the high-mobility group protein B1 [9]. Despite the heavy involvement of M1 macrophages in the processes that follow cardiac injury, the injured heart's macrophage population is typically heterogeneous in nature [9]. For example, using immunofluorescence, it was observed that M1 macrophages dominate the infarcted heart for the first three days but are later surpassed by M2 macrophages during days 5 through 7 $[2,3,13,20]$. Inflammation is then supposedly programmed to resolve when the cell debris is cleared $[20,21]$. Some have hypothesized that a downregulation of IL-6, TNF and matrix metalloproteinase (MMP)-9 is what allows for the overall macrophage population to shift into a preference for the reparative M2 phenotypes [7,12]. Moreover, it is speculated that one of the direct and intended consequences of this regulation is the appearance of specific cardiac macrophages that will trigger the processes of cardiac fibrosis and angiogenesis [7]. (Although is it hypothesized that a particular subset of macrophages is responsible for the initiation of these two processes, it is still ambiguous as to what their exact molecular profile is [7]). Note that murine models have demonstrated that the prolonged presence of M1 macrophages can lead to the expansion of infarct size and impede the resolution of inflammation and scar formation $[9,22]$.

Amongst the records identified for this review, accumulating evidence indicates that the heart's response to pathophysiological stress (i.e., in the face of a cardiac injury or cardiac disease) may be controlled by complex crosstalk between cardiomyocytes and macrophages [2,23]. It appears that the macrophage secretion of TNF- $\alpha$ has a direct, negative effect on cardiomyocyte contraction and myocardial contractility [12]. Through the utilization of the $\mathrm{CXCR}_{3} 1$ gene receptor in murine models, an extensive visualization of macrophage populations in the injured mouse heart has been made possible [13]. These systematic mappings revealed that in various seemingly strategic locations, abundant groupings of macrophages will cluster around the local cardiomyocytes. This was interpreted as an indication that macrophages occupy specific niches in the injured heart as a method of regulating the fate of cardiomyocytes [2,4]. Further transcriptional analysis suggested that the environment of these niches provide instructive signals that coordinate the local macrophage phenotype [4]. In addition to the previously described pieces of homeostatic evidence, this provides support in favour of the perspective that the cardiac environment plays a role in shaping the gene repertoire of macrophages and may even exert some control over macrophage-related functions [3]. Moreover, it is expected that alteration of these cardiac niches may affect the heart's network of resident macrophages.

\section{Macrophages in the Ageing Heart}

Although loosely defined, the "ageing heart" is typically in reference to one that is disease-free but, simply, due to the degradation of time has begun to show signs of inefficient performance. In general, ageing is greatly associated with an overall remodelling of the immune system [23]. Hearts, in particular, are shown to develop increased interstitial fibrosis, hypertrophy, and stiffness, which collectively contribute to increasing its susceptibility to disease and decreasing its capacity to efficiently pump blood $[9,24,25]$. Although multiple mechanisms have been proposed to explain how cardiac function changes during ageing, one of the most repeated narratives describes the alterations in the relative numbers of cardiac leukocytes as a primary trigger [9].

Evidence obtained using a custom-designed, quantitative PCR (qPCR) array to probe the expression levels of 45 target genes (associated with inflammation, angiogenesis, and fibrosis) in cardiomyocytes has demonstrated that the age-related decline in cardiac function is greatly associated with an increase in the production of inflammatory signals for monocyte recruitment [8]. The final conclusions also outlined that the ultimate decline in the self-renewal of cardiac resident macrophages is, in part, the most immediate cause (in a 
UNDERGRADUATE RESEARCH IN NATURAL AND CLINICAL SCIENCE AND TECHNOLOGY (URNCST) JOURNAL Read more URNCST Journal articles and submit your own today at: https://www.urncst.com

chain of causations) of cardiac ageing [9]. Laboratories have shown that there is a rapid decrease in antiinflammatory M2 macrophages and a linear increase in M1 macrophages over time [9,26]. This is because it is suspected that the declining population of cardiac resident macrophages is replenished by monocyte-derived macrophages, even in the absence of pathological triggers and inflammation [4]. Certain texts have also speculated that not a single cause may be responsible for this observed phenomenon; instead, it may be the combined result of exaggerated monocyte recruitment (in response to cardiac injury), alterations in monocyte fate specification or changes in resident macrophage behaviour [9,26].

Cardiac ageing is often accompanied by a range of byproduct symptoms and conditions, a hallmark being diastolic dysfunction. While diastolic dysfunction develops, cardiac macrophages produce IL-10, activate fibroblasts, and stimulate collagen deposition, thereby leading to impaired myocardial relaxation and increased myocardial stiffness [23]. Studies have shown that deletion of IL-10 in macrophages improves diastolic function [23]. Moreover, accompanying records have shown that MMP-9 is not only heavily involved in encouraging the vicious cycle of cardiac inflammation, but as it is solely responsible for activating young macrophages to an M1/M2 mid-transition phase. Thus, it is suspected that MMP-9 deletion may also prevent the decline in resident macrophage population $[6,9,23]$. Although much remains elusive about the exact relationship between macrophages and cardiac ageing, the basis of their proposed involvement lies on the idea that M1 macrophage polarization, in particular, is a driving contributor to the immuno-alterations of ageing [9,25].

\section{Discussion}

The renewed interest in cardioimmunology is primarily driven by the newly developed diagnostic abilities to obtain unbiased data with higher cellular resolution on cardiac cells that are so rare that, even a decade ago, they were generally assumed to be absent [6,9]. Applying techniques such as flow cytometry and intravital microscopy to the myocardium has provided new access to a world of cardiac immune cells [9]. However, despite the growing interest in cardiac macrophages, many questions, both broad and specific, remain unanswered. Some of these inquiries include, but are not limited to, how and why a relatively small number of cardiac macrophages appear to have such a significant influence over a great number of cardiac myocytes and fibroblasts; how various macrophage subsets communicate; and an extensive, systematic exploration of what kinds of stimulations trigger macrophage polarization $[7,12]$.

Most importantly, as a great majority of the extant literature makes use of murine models, a more in-depth translational analysis is required to describe how the data obtained from mice applies to humans [8]. The first studies of the human myocardium indicate that similar monocyte and macrophage subsets are involved, but further study of these cells could and should build a more substantial profile on their biological characteristics. In doing so, the doors to new options to treat acute cardiac injuries, heart failure and arrhythmias may widen [7].

Furthermore, several studies have concluded that the conversion between M1 and M2 macrophage phenotypes needs to be investigated systematically [9]. Certain studies specifically call for the mapping of macrophage phenotypes post-cardiac injury such that the turnover point from M1 macrophage dominance to M2 macrophage dominance can be narrowed down to a more specific time range [9]. This is so that the paradoxical nature of the heart's immunobehaviour may be better understood, thereby paving the way for further development in the treatment against cardiovascular disease and injuries (i.e., myocardial infarctions, cardiac ischemia, the cardiac symptoms of sepsis, etc.). Understanding the exact mechanisms at work in macrophage polarization also implies a step forward in identifying novel intervention targets for therapy [9]. Researchers have suggested that developing a computational model to mimic the macrophage continuum will help achieve this goal [9].

\section{Conclusions}

In conclusion, the works included in this review have established that like the brain, lung, spleen and intestines, the heart contains macrophages whose initial accumulation occurs early in development [3]. Cardiac macrophages are not tissue visitors, but essential, resident components to the heart [27]. As outlined in the results, the observed behaviour of cardiac macrophages in the steady state, injured and ageing heart have led to the development of three main perspectives on the biology of cardiac macrophages:

I. Macrophage ontogeny dictates function. Currently viewed as the strongest of the three arguments, this perspective relies on the idea that since macrophages originate from different physical locations and points in the development timeline, they are inherently responsible for different tasks. Evidence has demonstrated that distinct macrophage subsets may be identified via the expression intensity of various surface cell markers--many of which seem to also indicate their involvement in either the inflammatory or reparative processes.

II. The tissue dictates macrophage function. In this scenario, it is argued that the neighbouring cardiac tissue programs macrophage function regardless of its origins. Infiltrating monocytes may indeed contribute greatly to the process of inflammation, but that contribution may simply be numerical (as in the heart requires a greater number of macrophages than its resident macrophages to support the inflammatory process). Moreover, as 
UNDERGRADUATE RESEARCH IN NATURAL AND CLINICAL SCIENCE AND TECHNOLOGY (URNCST) JOURNAL Read more URNCST Journal articles and submit your own today at: https://www.urncst.com

macrophage polarization remains ambiguously defined, some have hypothesized that the various surface markers expressed by macrophages may fluctuate in expression intensity and do not necessarily consistently reflect their origins.

III. The nature versus nurture argument is a false division because the control of macrophages by ontogeny versus the control of macrophages by tissue environment are not mutually exclusive. In this scenario, infiltrating monocytes are akin to an on-demand buffer system that can be mobilized in times of crisis, but upon infiltration will listen to the programming of the surrounding tissue. This is so that the monocytes may integrate functionally and pursue the same tasks as the already present, resident macrophages. This scenario demonstrates that although their origins are imperative to the capabilities and survival of macrophage phenotypes, it is the neighbouring cardiac tissue that configures cellular function.

Nonetheless, from the design of new drugs and drug delivery systems to biomaterials and to tissue scaffolds, gaining a further understanding of the complexity of cardiac macrophages holds a promising future for therapeutic innovation [28].

\section{List of Abbreviations Used}

MHC-II: major histocompatibility complex class II

CCR2: CC chemokine receptor 2

Ly6C: lymphocyte antigen 6C

PRISMA: preferred reporting items for systematic review and meta-analyses

Mrc1: mannose receptor C-type 1

CD163: cluster of differentiation 163

Lyve-1: lymphatic vessel endothelial receptor 1

CXCL4: CXC chemokine receptor type 4

IL-4 : interleukine 4

IL-13 : interleukine 13

IL-1 $\beta$ : interleukin-1-beta

TGF- $\beta$ : transforming growth factor beta

AV: atrioventricular

QKI: quaking homolog

CACNA1C: calcium voltage-gated channel subunit

alpha1 C

MMP: matrix metalloproteinase

TNF- $\alpha$ : tumor necrosis factor alpha

\section{Conflicts of Interest}

The author(s) declare that they have no conflict of interests.

\section{Ethics Approval and/or Participant Consent}

This study did not require ethics approval or participant consent. It is presented as a systematic review and no primary data collection involving human patients was required.

\section{Authors' Contributions}

SC: contributed to the design of the study; collected and analyzed data; drafted the manuscript; and gave final approval of the version to be published.

\section{Acknowledgements}

I would like to thank Joseph Lee for his contributions in editing the manuscript and his guidance on the design of this study.

\section{Funding}

This study was not funded.

\section{References}

[1] Bajpai G, Schneider C, Wong N, Bredemeyer A, Hulsmans M, Nahrendorf M, Epelman S, Kreisel D, Liu Y, Itoh A, Shankar TS, Selzman CH, Drakos SG, Lavine KJ. The human heart contains distinct macrophage subsets with divergent origins and functions. Nature Medicine. 2018 Aug;24(8):1234-45. http://doi.org/10.1038/s41591-018-0059-x

[2] Gomez I, Duval V, Silvestre JS. Cardiomyocytes and macrophages discourse on the method to govern cardiac repair. Frontiers in Cardiovascular Medicine. 2018 Oct 2;5:314. http://doi.org/10.3389/fcvm.2018 .00134

[3] Swirski FK, Robbins CS, Nahrendorf M. Development and function of arterial and cardiac macrophages. Trends in Immunology. 2016;37(1):32-40. http://doi.org/10.1016/j.it.2015.11.004

[4] Epelman S, Lavine KJ, Beaudin AE, et al. Embryonic and adult-derived resident cardiac macrophages are maintained through distinct mechanisms at steady state and during inflammation. Immunity. 2014;40(1):91104. http://doi.org/10.1016/j.immuni.2013.11.019

[5] Leid J, Carrelha J, Boukarabila H, Epelman S, Jacobsen SEW, Lavine KJ. Primitive embryonic macrophages are required for coronary development and maturation. Circulation Research. 2016 May 13; 118(10):1498-511. http://doi.org/10.1161/ CIRCRESAHA. 115.308270

[6] Lafuse WP, Wozniak DJ, Rajaram MVS. Role of cardiac macrophages on cardiac inflammation, fibrosis and tissue repair. Cells. 2020 Dec 31;10(1):51. https://doi.org/10.3390/cells10010051

[7] Swirski, F.K., Nahrendorf, M. Cardioimmunology: The immune system in cardiac homeostasis and disease. Nature Reviews Immunology. 2018;18:733-744. https://doi.org/10.1038/s41577-018-0065-8

[8] Ramos GC, van den Berg A, Nunes-Silva V, et al. Myocardial aging as a T-cell-mediated phenomenon. Proceedings of the National Academy of Sciences of the United States of America. $2017 \mathrm{Mar}$ 2;114(12):E2420-E2429. https://doi.org/10.1073/pnas .1621047114 
UNDERGRADUATE RESEARCH IN NATURAL AND CLINICAL SCIENCE AND TECHNOLOGY (URNCST) JOURNAL Read more URNCST Journal articles and submit your own today at: https://www.urncst.com

[9] Ma Y, Mouton AJ, Lindsey ML. Cardiac macrophage biology in the steady-state heart, the ageing heart, and following myocardial infarction. Translational Research. 2018;191:15-28. https://doi.org/10.1016/ j.trsl.2017.10.001

[10] Swirski FK, Robbins CS, Nahrendorf M. Development and function of arterial and cardiac macrophages. Trends in Immunology. 2016;37(1):32-40. http://doi.org/10.1016/j.it.2015.11.004

[11] Pinto AR, Paolicelli R, Salimova E, Gospocic J, Slonimsky E, Bilbao-Cortes D, Godwin JW, Rosenthal NA. An abundant tissue macrophage population in the adult murine heart with a distinct alternatively activated macrophage profile. Public Library of Science One. 2012;7(5):e36814. https://doi.org/10.1371/journal.pone .0036814

[12] Katsuhito Fujiu, Jack Wang, Ryozo Nagai, Cardioprotective function of cardiac macrophages. Cardiovascular Research. 2014 May 1;102(2):232-9. https://doi.org/10.1093/cvr/cvu059

[13] Lavine KJ, Epelman S, Uchida K, Weber KJ, Nichols CG, Schilling JD, Ornitz DM, Randolph, GJ, Mann DL. Distinct macrophage lineages contribute to disparate patterns of cardiac recovery and remodelling in the neonatal and adult heart. Proceedings of the National Academy of Sciences of the United States of America. 2014 Nov 11;111(45):16029-34. https://doi.org/10.1073/pnas.1406508111

[14] Gordon S, Martinez FO. Alternative activation of macrophages: Mechanism and functions. Immunity. 2010 May 28;32(5):593-604. http://doi.org/10.1016/ j.immuni.2010.05.007

[15] Falkenham A, Myers T, Wong C, Legare JF. Implications for the role of macrophages in a model of myocardial fibrosis: CCR2 (-/-) mice exhibit an M2 phenotypic shift in resident cardiac macrophages. Cardiovascular Pathology. 2016 Oct;25(5):390-8. https://doi.org/10.1016/j.carpath.2016.05.006

[16] Ensan S, Li A, Besla R, Degousee N, Cosme J, Roufaiel M, Shikatani EA, El-Maklizi M, Williams JW, Robins L, Li C, Lewis B, Yun TJ, Lee JS, Wieghofer P, Khattar R, Farrokhi K, Byrne J, Ouzounian M, Zavitz CC, Levy GA, Bauer CM, Libby P, Husain M, Swirski FK, Cheong C, Prinz M, Hilgendorf I, Randolph GJ, Epelman S, Gramolini AO, Cybulsky MI, Rubin BB, Robbins CS. Self-renewing resident arterial macrophages arise from embryonic CX3CR1(+) precursors and circulating monocytes immediately after birth. Nature Immunology. 2016 Feb;17(2):159-68. https://doi.org/10.1038/ni.3343

[17] Sun Z, Zhou D, Xie X. et al. Crosstalk between macrophages and atrial myocytes in atrial fibrillation. Basic Research in Cardiology. 2016 Mar 26;111:63. https://doi.org/10.1007/s00395-016-0584-Z
[18] Oishi S, Sasano T, Tateishi Y, Tamura N, Isobe M, Furukawa T. Stretch of atrial myocytes stimulates recruitment of macrophages via ATP release through gap-junction channels. Journal of Pharmaceutical Sciences. 2012; 120(4):296-304. https://doi.org/ 10.1254/jphs.12202FP

[19] Frantz S, Nahrendorf M. Cardiac macrophages and their role in ischaemic heart disease. Cardiovascular Research. 2014;102(2):240-8. https://doi.org/10.1093/ $\mathrm{cvr} / \mathrm{cvu} 025$

[20] Frangogiannis, N. The inflammatory response in myocardial injury, repair, and remodelling. Nature Reviews Cardiology. 2014 Mar 15;11:255-65. https://doi.org/10.1038/nrcardio.2014.28

[21] Lai, SL., Marín-Juez, R. \& Stainier, D.Y.R. Immune responses in cardiac repair and regeneration: A comparative point of view. Cellular and Molecular Life Sciences. 2019 Apr 15;76:1365-80. https://doi.org/ 10.1007/s00018-018-2995-5

[22] Heidt T, Courties G, Dutta P, Sager HB, Sebas M, Iwamoto Y, Sun Y, Da Silva N, Panizzi P, van der Laan AM, Swirski FK, Weissleder R, Nahrendorf M. Differential contribution of monocytes to heart macrophages in steady-state and after myocardial infarction. Circulation Research. 2014 Jul 7;115(2):28495. https://doi.org/10.1161/CIRCRESAHA.115.303567

[23] Hulsmans M, Sager HB, Roh JD, Valero-Muñoz M, Houstis NE, Iwamoto Y, Sun Y, Wilson RM, Wojtkiewicz G, Tricot B, Osborne MT, Hung J, Vinegoni C, Naxerova K, Sosnovik DE, Zile MR, Bradshaw AD, Liao R, Tawakol A, Weissleder R, Rosenzweig A, Swirski FK, Sam F, Nahrendorf M. Cardiac macrophages promote diastolic dysfunction. Journal of Experimental Medicine. $2018 \mathrm{Feb}$ 5;215(2):423-40. https://doi.org/10.1084/jem.20171274

[24] Sugita J, Fujiu K, Nakayama Y, Matsubara T, Matsuda J, Oshima T, Liu Y, Maru Y, Hasumi E, Kojima T, Seno H, Asano K, Ishijima A, Tomii N, Yamazaki M, Kudo F, Sakuma I, Nagai R, Manabe I, Komuro I. Cardiac macrophages prevent sudden death during heart stress. Nature Communications. $2021 \mathrm{Mar}$ 26;12(1):1910. https://doi.org/10.1038/s41467-02122178-0

[25] Molawi K, Wolf Y, Kandalla PK, Favret J, Hagemeyer N, Frenzel K, Pinto AR, Klapproth K, Henri S, Malissen B, Rodewald HR, Rosenthal NA, Bajenoff M, Prinz M, Jung S, Sieweke MH. Progressive replacement of embryo-derived cardiac macrophages with age. Journal of Experimental Medicine. 2014 Oct 20;211(11):2151-8. https://doi.org/10.1084/jem.20140639

[26] Zhang S, Chen R, Chakrabarti S, Su Z. Resident macrophages as potential therapeutic targets for cardiac ageing and injury. Clinical \& Translational Immunology. 2020 Aug 26;9(8):e1167. https://doi.org/ $\underline{10.1002 / \mathrm{cti} 2.1167}$ 
UNDERGRADUATE RESEARCH IN NATURAL AND CLINICAL SCIENCE AND TECHNOLOGY (URNCST) JOURNAL Read more URNCST Journal articles and submit your own today at: https://www.urncst.com

[27] Jain N, Moeller J, Vogel V. Mechanobiology of macrophages: How physical factors coregulate macrophage plasticity and phagocytosis. Annual Review of Biomedical Engineering. 2019;21:267-97. https://doi.org/10.1146/annurev-bioeng-062117-121224
[28] Chen M, Li X, Wang S, Yu L, Tang J and Zhou S. The role of cardiac macrophage and cytokines on ventricular arrhythmias. Frontiers in Physiology. 2020;11:1113. https://doi.org/10.3389/fphys.2020 $\underline{.01113}$

\section{Article Information}

Managing Editor: Jeremy Y. Ng

Peer Reviewers: Joseph Lee, Foram Vyas

Article Dates: Received Aug 05 21; Accepted Oct 14 21; Published Oct 2821

\section{Citation}

Please cite this article as follows:

Cai S. The behaviour of cardiac macrophages in the steady state, injured, and ageing heart: A systematic review. URNCST Journal. 2021 Oct 28: 5(10). https://urncst.com/index.php/urncst/article/view/307

DOI Link: https://doi.org/10.26685/urncst.307

\section{Copyright}

(C) Susan Cai. (2021). Published first in the Undergraduate Research in Natural and Clinical Science and Technology (URNCST) Journal. This is an open access article distributed under the terms of the Creative Commons Attribution License (https://creativecommons.org/licenses/by/4.0/), which permits unrestricted use, distribution, and reproduction in any medium, provided the original work, first published in the Undergraduate Research in Natural and Clinical Science and Technology (URNCST) Journal, is properly cited. The complete bibliographic information, a link to the original publication on http://www.urncst.com, as well as this copyright and license information must be included.

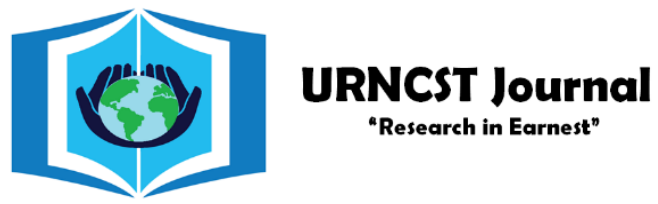

\section{Funded by the Government of Canada}

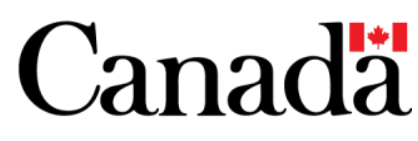

Do you research in earnest? Submit your next undergraduate research article to the URNCST Journal!

| Open Access | Peer-Reviewed | Rapid Turnaround Time | International | | Broad and Multidisciplinary | Indexed | Innovative | Social Media Promoted |

Pre-submission inquiries? Send us an email at info@ urncst.com | Facebook, Twitter and LinkedIn: @URNCST

Submit YOUR manuscript today at https://www.urncst.com! 